\title{
Uniqueness theorem for stationary axisymmetric black holes in Einstein-Maxwell-axion-dilaton gravity
}

\author{
Marek Rogatko \\ Institute of Physics \\ Maria Curie-Sklodowska University \\ 20-031 Lublin, pl. Marii Curie-Sklodowskiej 1, Poland \\ rogat@kft.umcs.lublin.pl \\ marek.rogatko@poczta.umcs.lublin.pl
}

(Dated: October 26, 2018)

\begin{abstract}
We prove the uniqueness theorem for stationary axisymmetric black holes solution in EinsteinMaxwell-axion-dilaton gravity being the low-energy limit of the heterotic string theory. We consider both the non-extremal and extremal Kerr-Sen black hole solutions.

PACS numbers: 04.70.Bw
\end{abstract}

\section{INTRODUCTION}

Much efforts have been devoted to the studies of the most striking investigations related to the black hole equilibrium states, the uniqueness theorem in four-dimensional spacetime. The pioneering investigations were presented by Israel in Refs. [1, 2]. Müller zum Hagen et al. [3] and Robinson [4] were able to find the generalization of Israel's theorems. It was shown that Schwarzschild and Reissner-Nordström (RN) solutions were the only Einstein or Einstein-Maxwell (EM) (non-extreme) solutions that satisfied the conditions of being static black hole metrics. Quite different approach to the aforementioned problem using a conformal transformation on a spacelike hypersurface and the positive mass theorem [5, 6] was proposed in [7]-[10]. The complete classification of four-dimensional vacuum black holes was established in Ref.[11, 12], where the condition of non-degeneracy of the event horizon was removed. Studies of the near-horizon geometry conducted in Ref.[13] enabled to eliminate the last restrictive condition of the static electrovacuum no-hair theorem.

The turning point for establishing the uniqueness of stationary and axisymmetric black hole spacetimes being the solution of vacuum Einstein equations were achieved by Carter [14, 15] and Robinson [16. The systematic way of obtaining the desire results in electromagnetic case was presented by Mazur [17, 18] and Bunting [19] (see also Ref.[20]). For a review of the uniqueness of black holes solutions story in four-dimensions see, e.g, [18] and [21].

Nowadays, there has been also an active period of constructing black hole solutions in the string theories (see [22] and references therein). The stationary axisymmetric black hole solution in four-dimensional Einstein-Maxwellaxion-dilaton (EMAD) gravity being the effective theory of the heterotic string theory was obtained by Sen 23]. The $\sigma$-model representation and symmetries of stationary axisymmetric solutions in EMAD gravity were widely studied [24]-33]. One should also mention the efforts of proving the uniqueness theorem for the black holes appearing in the theory in question [34]-[40].

Recently, the uniqueness theorem for the extremal black hole solutions acquired much attention. It was shown that the near-horizon geometry of any extremal vacuum black hole must agree with the extremal Kerr black hole line element [41]. The same statement is also valid in the case of Kerr-Newman spacetime. The uniqueness for asymptotically flat stationary extremal vacuum black hole solutions in four and five-spacetime dimensions were presented in Ref. 42]. Using the Mazur identity it was proved that the only four-dimensional stationary axisymmetric asymptotically flat vacuum black hole with a single degenerate horizon was extremal Kerr solution or extremal Kerr-Newman in the case of EM theory [43]. The different method of proving the uniqueness theorem for extremal Kerr black hole was proposed in Ref. [44] On the other hand, mathematically rigorous proof of the uniqueness theorem for the extremal Kerr-Newman black hole was elaborated in Ref. 45].

In our paper we shall consider the stationary axisymmetric black hole solutions to the EMAD gravity (Kerr-Sen solution) using $\sigma$-model representation of the underlying theory. We shall take into account both the non-degenerate as well as the extremal Kerr-Sen black holes. We prove the uniqueness theorem for both stationary axisymmetric black holes in this theory. It enables us to close a gap that existed for many years in the proof of the uniqueness theorem for black holes being the solutions of the low-energy effective heterotic string theory.

Our paper is organized as follows. In Sec.II we review the $\sigma$-model representation for the field equations of EMAD gravity being the low-energy limit of the heterotic string theory. This effective theory comprises gravitational field $g_{\mu \nu}$, scalar field dilaton, the $U(1)$-gauge field and the Kalb-Ramond antisymmetric tensor field which is equivalent in four-dimensional spacetime to the Peccei-Quinn antisymmetric pseudo-scalar axion. The next subsection will be devoted to the Mazur identity, the main tool for proving the uniqueness theory for stationary axisymmetric black 
hole in the theory in question. In subsection $\mathrm{C}$ we determine the boundary conditions and show that two stationary axisymmetric solutions of EMAD gravity subject to the same boundary and regularity conditions coincide with each other. In Sec.III the near-horizon geometry of the stationary axisymmetric extremal solution to EMAD-gravity and the boundary conditions for the fields appearing in the theory will be analyzed. All these help us to to find the uniqueness theorem for the extremal Kerr-Sen black hole solution.

\section{UNIQUENESS THEOREM FOR KERR-SEN SOLUTION}

\section{A. $\sigma$-model representation for EMAD-gravity}

In this subsection we review the derivation of the three-dimensional $\sigma$-model representation for the bosonic part of a heterotic string theory compactified to four-dimensions. The so-called EMAD gravity contains metric tensor $g_{\mu \nu}$, $U(1)$ gauge field, the Kalb-Ramond antisymmetric tensor $B_{\alpha \beta}$ and dilaton field $\phi$. In four-dimensional spacetime the Kalb-Ramond tensor is equivalent to the pseudo-scalar axion field. The resulting effective action for the bosonic sector of the heterotic string with one gauge field is provided by

$$
S=\int d^{4} x \sqrt{-g}\left(R-2 \nabla^{\mu} \phi \nabla_{\mu} \phi-\frac{1}{2} e^{4 \phi} \nabla^{\mu} a \nabla_{\mu}-e^{-2 \phi} F_{\mu \nu} F^{\mu \nu}-a F_{\mu \nu} * F^{\mu \nu}\right),
$$

where $* F_{\mu \nu}=1 / 2 \epsilon_{\mu \nu \rho \gamma} F^{\rho \gamma}$. In order to reduce the system under consideration to three-dimensions one should have a non-zero Killing vector field. Introducing the timelike Killing vector field allows us to rewrite a metric for an arbitrary stationary configuration in the form as [4]

$$
d s^{2}=-f\left(d t-\omega_{a} d x^{a}\right)^{2}+\frac{h_{i j}}{f} d x^{i} d x^{j}
$$

where $h_{i j}$ is three-dimensional metric, $\omega_{a}$ is the rotation vector, while $f$ is scalar. All the line coefficients depend on $x^{i}$, where $i=1, \ldots 3$.

Due to the existence of timelike Killing vector field it will be possible to decompose the generalized Maxwell equations into the two components field, electric and magnetic. Namely, one has respectively the following relations [24]-[26]:

$$
\begin{gathered}
F_{i 0}=\frac{1}{\sqrt{2}} \partial_{i} v \\
e^{-2 \phi} F^{a b}+a * F^{a b}=\frac{f}{\sqrt{2 h}} \epsilon^{a b c} \partial_{c} \kappa
\end{gathered}
$$

where $v$ is the electric potential, while $\kappa$ is responsible for the magnetic one. Further, one can introduce the torsion vector [46] defined as

$$
\tau^{m}=-\frac{f^{2}}{\sqrt{h}} \epsilon^{m j k} \partial_{j} \omega_{k}
$$

It turned out that it can be rewritten by means of the twist potential $\chi$ in the sense defined in Ref. 46]

$$
\tau_{i}=\partial_{i} \chi+v \partial_{i} \kappa-\kappa \partial_{i} v
$$

The most important feature of the above EMAD-gravity equations of motion is the fact that all the above relations can be achieved by variation of the action provided by [29, 33]

$$
\begin{aligned}
S & =\int d^{3} x \sqrt{h}\left({ }^{3} R+\frac{1}{2 f^{2}}\left(\partial_{i} f \partial^{i} f+\tau_{i} \tau^{i}\right)-2 \partial_{m} \phi \partial^{m} \phi-\frac{1}{2} e^{4 \phi} \partial_{m} a \partial^{m} a\right. \\
& \left.+\frac{1}{f}\left(e^{2 \phi}\left(\partial_{i} \kappa-a \partial_{i} v\right)\left(\partial^{i} \kappa-a \partial^{i} v\right)+e^{-2 \phi} \partial_{k} v \partial^{k} v\right)\right) .
\end{aligned}
$$

Consequently, it can be proved that the above equations can be cast into a set of relations derived from the action for $\sigma$-model for vector potential $\Phi_{A}$ coupled to three-dimensional gravity. Namely, the action is provided by [27]

$$
S=\int d^{3} x \sqrt{h}\left({ }^{(3)} R-G_{A B} \partial_{i} \Phi^{A} \partial_{j} \Phi^{B} h^{i j}\right),
$$


where the line element of the target space implies

$$
\begin{aligned}
G_{A B} d \Phi^{A} d \Phi^{B} & =\frac{1}{2} e^{2(\eta-\zeta)} d a^{2}+d \eta^{2}+d \zeta^{2}-e^{2 \zeta} d v^{2}-e^{2 \eta}(d \kappa-a d v)^{2} \\
& +\frac{1}{2} e^{2(\eta-\zeta)}(d \chi+v d \kappa-\kappa d v)^{2}
\end{aligned}
$$

where we have denoted $\eta=\phi-1 / 2 \ln f$ and $\zeta=-(\phi+1 / 2 \ln f)$. The vector potential $\Phi_{A}$ has six non-zero components $(f, v, \kappa, \chi, a, \phi)$. From the action (8) we can get the standard equations of motion for gravitating $\sigma$-model. They imply

$$
{ }^{(3)} R=G_{A B} \partial_{i} \Phi^{A} \partial_{j} \Phi^{B} h^{i j} .
$$

Varying the action (8) with respect to $\Phi_{A}$ we arrive at the equation of motion for $\Phi^{A}$ field

$$
{ }^{(h)} \nabla_{i}{ }^{(h)} \nabla^{i} \Phi^{A}+h^{i j} \Gamma_{B C}^{A} \partial_{i} \Phi^{B} \partial_{j} \Phi^{C}=0
$$

where ${ }^{(h)} \nabla_{i}$ is the nabla operator with respect to three-dimensional metric $h_{i j}$, while $\Gamma^{A}{ }_{B C}$ is the Christoffel symbol in the target space metric $G_{A B}$.

Now, we proceed to consider another Killing vector field which is assumed to commute with the timelike one introduced before. It implies, without loss of generality, that the three-dimensional metric can be expressed as follows:

$$
d h^{2}=e^{2 \gamma}\left(d \rho^{2}+d z^{2}\right)+\rho^{2} d \phi^{2},
$$

Hence, the equation of motion for $\Phi_{A}$ field yields

$$
{ }^{(h)} \nabla_{i}{ }^{(h)} \nabla^{i} \Phi^{A}+\Gamma_{B C}^{A}\left(\partial_{\rho} \Phi^{B} \partial_{\rho} \Phi^{C}+\partial_{z} \Phi^{B} \partial_{z} \Phi^{C}\right)=0 .
$$

On this account, the function $\gamma$ may be determined by the equations

$$
\begin{aligned}
& { }^{(h)} R_{\rho \rho}-{ }^{(h)} R_{z z}=\frac{2 \partial_{r} \gamma}{\rho}=G_{A B}\left(\partial_{\rho} \Phi^{A} \partial_{\rho} \Phi^{B}+\partial_{z} \Phi^{A} \partial_{z} \Phi^{B}\right), \\
& { }^{(h)} R_{\rho z}=\frac{\partial_{z} \gamma}{\rho}=G_{A B} \partial_{\rho} \Phi^{A} \partial_{z} \Phi^{B} .
\end{aligned}
$$

Then, the action of the system can be rewritten in terms of the current matrix $J^{i}[29$, 31, 32]

$$
S=\frac{1}{4} \int d \rho d z \rho \operatorname{Tr}\left(J^{i} J_{i}\right)
$$

where $J^{i}=\nabla^{i} M M^{-1}$. Using the Gauss decomposition, the symmetric matrix $M$ may be written as

$$
M=\left(\begin{array}{cc}
P^{-1} & P^{-1} Q \\
Q P^{-1} & P+Q P^{-1} Q
\end{array}\right)
$$

while symmetric two-dimensional matrices $P$ and $Q$ are given by the following:

$$
P=\left(\begin{array}{cc}
f-e^{-2 \phi} v^{2} & -e^{-2 \phi} v \\
-e^{-2 \phi} v & -e^{-2 \phi}
\end{array}\right), \quad Q=\left(\begin{array}{cc}
-\chi+v w & w \\
w & -a
\end{array}\right)
$$

where we have denoted $w=\kappa-a v$.

\section{B. Mazur identity}

In the proof of the uniqueness theorem for stationary axisymmetric black hole solution in EMAD gravity a key role will be played by the so-called Mazur identity [17, 21]. Let us consider two sets of field configurations $M_{[0]}$ and $M_{[1]}$ 
satisfying the equation of motion of the underlying theory and denote the difference $J_{\text {diff }}$ between those two field configurations by the following relation:

$$
J_{\text {diff }}^{i}=J_{[1]}^{i}-J_{[0]}^{i}=\nabla^{i} M_{[1]} M_{[1]}^{-1}-\nabla^{i} M_{[0]} M_{[0]}^{-1} .
$$

Then, we define the deviation matrix $\Psi$ which implies

$$
\Psi=M_{\text {diff }} M_{[0]}^{-1}=M_{[1]} M_{[0]}^{-1}-\mathbf{1},
$$

where 1 is the unit matrix and $M_{\text {diff }}=M_{[1]}-M_{[0]}$. One can remark that the deviation matrix $\Psi$ will be equal to zero matrix if and only if the two field configurations will accord. Expressing the Laplacian of the deviation matrix in the manner of $\rho$ and $z$-coordinates one arrives at the following:

$$
\partial_{i}\left(\rho \partial^{i} \operatorname{tr} \Psi\right)=\rho h_{i j}\left(J_{i \text { diff }}^{t} M_{[0]}^{-1} J_{d i f f}^{i} M_{[1]}\right),
$$

where we define the transpose current matrix in the form as follows:

$$
J_{i}^{t}=M^{-1} \nabla_{i} M
$$

By integrating the above equation over the adequate region $\Omega$ of the $(\rho, z)$-plane and by means of the Green theorem we get the expression

$$
\int_{\partial \Omega} \rho \partial^{m}(\operatorname{tr} \Psi) d S_{m}=\int_{\Omega} \rho h_{i j}\left(J_{i \text { diff }}^{t} M_{[0]}^{-1} J_{d i f f}^{i} M_{[1]}\right) d \rho d z .
$$

The boundary of the region in question embraces the black hole event horizon, plane of rotation and the infinity. Because of the fact that matrix $M$ has a square root matrix $m$, i.e., $M=m m^{t}$ one can rewrite the Mazur identity in a suitable form. On this account, one arrives at

$$
\int_{\partial \Omega} \rho \partial^{m}(\operatorname{tr} \Psi) d S_{m}=\int_{\Omega} \rho h_{i j}\left(\operatorname{tr} \Phi_{i}^{t} \Phi^{i}\right) d \rho d z
$$

where $\Phi_{k}=m_{[1]} J_{k \text { diff }}^{t} m_{[0]}^{-1}$. It can be seen that the right-hand side of Eq.(24) is non-negative. If one impose the boundary conditions on $\partial \Omega$ such that the left-hand side of the relation disappears, we can conclude that $J_{d i f f}^{i}=0$. From the relation $\nabla^{i} \Psi=M_{[0]}^{-1} J_{d i f f}^{i} M_{[1]}$ it also follows that the matrix $\Psi$ has to be constant over the considered region $\Omega$. In particular, if the matrix in question is equal to zero matrix, then it yields that two solutions $M_{[0]}$ and $M_{[1]}$, subject to the same boundary and regularity conditions match each other.

\section{Boundary conditions}

In this subsection we shall apply the Mazur identity for the field configuration described by the $c u r r e n t$ matrix $J_{i}$. We shall look for the boundary conditions of the fields at infinity, on the plane of rotation as well as at the black hole event horizon. Moreover, we assume asymptotical flatness of the considered black hole solution to EMAD gravity and regularity on a plane of rotation and on the the black hole event horizon.

The Kerr-Sen metric being the stationary axisymmetric solution to EMAD gravity was derived in Ref.[23]. The metric of the Kerr-Sen black hole spacetime implies

$$
d s^{2}=-\frac{\Delta}{\Sigma}\left(d \hat{t}-a \sin ^{2} \theta d \hat{\phi}\right)^{2}+\frac{\sin ^{2} \theta}{\Sigma}\left[\left(\hat{r}\left(\hat{r}-r_{-}\right)+a^{2}\right) d \hat{\phi}-a d \hat{t}\right]^{2}+\frac{\Sigma}{\Delta} d \hat{r}^{2}+\Sigma d \theta^{2}
$$

where we have defined the following quantities:

$$
\Sigma=\hat{r}\left(\hat{r}-r_{-}\right)+a^{2} \cos ^{2} \theta, \quad \Delta=\left(\hat{r}-r_{-}\right)(\hat{r}-2 M)+a^{2}, \quad r_{-}=\frac{Q^{2}}{M} .
$$

$M$ is the black hole mass, $Q$ is attributed to its charge, while $a$ is the Kerr rotation parameter related to the black hole angular momentum by $J=a M$. The black hole event horizon radii are located at

$$
\hat{r}_{ \pm}=M+\frac{r_{-}}{2} \pm \sqrt{\left(M-\frac{r_{-}}{2}\right)^{2}-a^{2}}
$$


Furthermore, the asymptotic behaviour of the metric coefficients are provided by relations

$$
\begin{array}{rlrl}
g_{\hat{t} \hat{t}} & =-\left(1-\frac{2 M}{\hat{r}}+\mathcal{O}\left(\frac{1}{\hat{r}^{2}}\right)\right), & g_{\hat{t} \hat{\phi}}=-\frac{2 J \sin ^{2} \theta}{\hat{r}}+\mathcal{O}\left(\frac{1}{\hat{r}^{2}}\right), \\
g_{\hat{\phi} \hat{\phi}}=\left[\hat{r}\left(\hat{r}-r_{-}\right)+\mathcal{O}(\hat{r})\right] \sin ^{2} \theta, & g_{\theta \theta}=\hat{r}\left(\hat{r}-r_{-}\right)+\mathcal{O}(\hat{r}),
\end{array}
$$

where $M$ and $J$ are respectively mass and angular momentum asymptotically conserved at spherical spatial infinity. For the fields existing in the theory under consideration, i.e., for dilaton field, electric and magnetic part of $U(1)$ gauge field and for the axion one, we have the following limits as $\hat{r}$ tends to infinity:

$$
\phi \simeq \mathcal{O}\left(\frac{1}{\hat{r}}\right), \quad v \simeq \mathcal{O}\left(\frac{1}{\hat{r}}\right), \quad \kappa \simeq \mathcal{O}\left(\frac{1}{\hat{r}^{2}}\right), \quad a \simeq \mathcal{O}\left(\frac{1}{\hat{r}^{2}}\right)
$$

On the other hand, the fields existing in in the theory under consideration, i.e., dilaton and axion fields and electric and magnetic part of $U(1)$ gauge field and for the axion one, yield

$$
\begin{aligned}
e^{2 \phi} & =\frac{\left(\hat{r}-r_{-}\right)^{2}+a_{k}^{2} \cos ^{2} \theta}{\Sigma}, & a & =\frac{r_{-} a_{k} \cos \theta}{\left(\hat{r}-r_{-}\right)^{2}+a_{k}^{2} \cos ^{2} \theta}, \\
v & =\left(2 M r_{-}\right)^{-\frac{1}{2}}\left(\hat{r}-r_{-}\right) \Sigma, & \kappa & =\frac{r_{-} a_{k} \cos \theta}{\Sigma} .
\end{aligned}
$$

They have the following limits as $r$ tends to infinity:

$$
\phi \simeq \mathcal{O}\left(\frac{1}{\hat{r}}\right), \quad v \simeq \mathcal{O}\left(\frac{1}{\hat{r}}\right), \quad \kappa \simeq \mathcal{O}\left(\frac{1}{\hat{r}^{2}}\right), \quad a \simeq \mathcal{O}\left(\frac{1}{\hat{r}^{2}}\right)
$$

As in Ref. 47], comparing the asymptotic forms of the metric tensor coefficients given by relations (28) with the WeylPapapetrou metric (2), we derive the boundary conditions. On the other hand, one can assume that they behave in some manner and conduct the uniqueness proof [48].

Moreover, the regularity conditions on the rotation plain requires that $g_{\phi \phi}$ should have the form $g_{\phi \phi}=f_{\phi \phi} \sin ^{2} \theta$. It implies that $f_{\phi \phi} \simeq \hat{r}\left(\hat{r}-r_{-}\right)+\mathcal{O}(\hat{r})$.

The event horizon has $S^{2}$-topology which enables one to introduce the spheroidal coordinates

$$
z=\lambda \mu, \quad \rho^{2}=\left(\lambda^{2}-c^{2}\right)\left(1-\mu^{2}\right),
$$

where $\mu=\cos 2 \theta$. The boundary $\lambda=c$ is responsible for the black hole event horizon, while two rotation axis segments distinguishing the north and south of the horizon are given by the respective limit $\mu= \pm 1$. Consequently, the asymptotic behaviour of $f_{\phi \phi}$ implies

$$
\begin{aligned}
& \mu \rightarrow \pm 1 \Rightarrow f_{\phi \phi} \simeq \mathcal{O}(1), \quad \lambda \rightarrow c \Rightarrow f_{\phi \phi} \simeq \mathcal{O}(1), \\
& \lambda \rightarrow \infty \Rightarrow f_{\phi \phi} \simeq \lambda+\frac{r_{-}}{2} \sqrt{\lambda}-\frac{r_{-}^{2}}{4}+\mathcal{O}\left(\lambda^{-\frac{1}{2}}\right) .
\end{aligned}
$$

Similarly, one has that

$$
g_{\phi \phi}=\frac{\rho^{2}}{f}-f \omega^{2}=\left[\hat{r}\left(\hat{r}-r_{-}\right)+\mathcal{O}(\hat{r})\right] \sin ^{2} \theta
$$

where $f=f_{\phi \phi} \sin ^{2} \theta$. It may be noted that $\rho$ is provided by the following relation:

$$
\rho^{2}=\left[\hat{r}^{2}\left(\hat{r}-r_{-}\right)^{2}+\mathcal{O}(\hat{r})\right] \sin ^{2} \theta
$$

By virtue of the above, it can be seen that $\rho$ vanishes at the $\phi$-invariant plane, where $\sin \theta=0$, and also on the event horizon due to the form of the metric (2). It is also clear from the definition of $\lambda$, that the relation between $\lambda$ and $\hat{r}$ yields

$$
\lambda=\hat{r}\left(\hat{r}-r_{-}\right)+\mathcal{O}\left(\frac{1}{\hat{r}}\right)
$$


while for $r$ as a function of $\lambda$, we get

$$
\hat{r}=\sqrt{\lambda}\left(1-\frac{r_{-}}{8 \lambda}+\mathcal{O}\left(\frac{1}{\hat{r}}\right)\right)+\frac{r_{-}}{2} .
$$

It follows directly that, the asymptotic behaviours of $\rho$ are determined by the formulae

$$
\begin{aligned}
& \mu \rightarrow 1 \Rightarrow \rho \simeq \mathcal{O}(\sqrt{1-\mu}), \\
& \mu \rightarrow-1 \Rightarrow \rho \simeq \mathcal{O}(\sqrt{1+\mu}), \\
& \lambda \rightarrow c \Rightarrow \rho \simeq \mathcal{O}(\sqrt{\lambda-c}), \\
& \lambda \rightarrow \infty \Rightarrow \rho \simeq \mathcal{O}(\lambda),
\end{aligned}
$$

On the other hand, the following form of equation may be noted for the rotation vector:

$$
\omega_{\phi}=-\frac{2 J}{\hat{r}^{2}\left(\hat{r}-r_{-}\right)}+\mathcal{O}\left(\hat{r}^{-5}\right)
$$

As usual we take into account the domain of outer communication $\langle<\mathcal{J}>>$ as an oriented rectangle. Namely, one has the following:

$$
\begin{aligned}
\partial \mathcal{J}^{(1)} & =\{\mu=1, \lambda=c, \ldots, R\}, \\
\partial \mathcal{J}^{(2)} & =\{\lambda=c, \mu=1, \ldots,-1\}, \\
\partial \mathcal{J}^{(3)} & =\{\mu=-1, \lambda=c, \ldots, R\}, \\
\partial \mathcal{J}^{(4)} & =\{\lambda=R, \mu=-1, \ldots, 1\} .
\end{aligned}
$$

The corresponding metric on the domain of outer communication $\langle<\mathcal{J}\rangle>$, written in spheroidal coordinates is given by

$$
d s_{<<\mathcal{J}>>}^{2}=\left(\lambda^{2}-c^{2} \mu^{2}\right)\left(\frac{d \lambda^{2}}{\lambda^{2}-c^{2}}+\frac{d \mu^{2}}{1-\mu^{2}}\right) .
$$

Then, the boundary integral on the left-hand side of the Mazur identity (24) may be written as

$$
\int_{\partial \mathcal{J}} \rho \partial^{a}(\operatorname{tr} \Psi) d S_{a}=\int_{\partial \mathcal{J}} d \lambda \rho \sqrt{\frac{h_{\lambda \lambda}}{h_{\mu \mu}}} \partial_{\mu}(t r \Psi)-\int_{\partial \mathcal{J}} d \mu \rho \sqrt{\frac{h_{\mu \mu}}{h_{\lambda \lambda}}} \partial_{\lambda}(t r \Psi) .
$$

We can also readily write down the asymptotic forms of $\sqrt{h_{\lambda \lambda} / h_{\mu \mu}}$ and $\sqrt{h_{\mu \mu} / h_{\lambda \lambda}}$. They become respectively

$$
\mu \rightarrow 1 \Rightarrow \sqrt{\frac{h_{\lambda \lambda}}{h_{\mu \mu}}} \rightarrow \mathcal{O}(\sqrt{1-\mu}), \quad \mu \rightarrow-1 \Rightarrow \sqrt{\frac{h_{\lambda \lambda}}{h_{\mu \mu}}} \rightarrow \mathcal{O}(\sqrt{1+\mu}),
$$

and similarly we achieve

$$
\lambda \rightarrow c \Rightarrow \sqrt{\frac{h_{\mu \mu}}{h_{\lambda \lambda}}} \rightarrow \mathcal{O}(\sqrt{\lambda-c}), \quad \lambda \rightarrow \infty \Rightarrow \sqrt{\frac{h_{\mu \mu}}{h_{\lambda \lambda}}} \rightarrow \mathcal{O}(\lambda) .
$$

In what follows we have used MAPLE symbolic mathematical program to calculate the trace of deviation matrix.

First let us consider the behaviour of the deviation matrix near axes. From Eqs.(39) and (40) we see that $\rho \rightarrow$ $\mathcal{O}(\sqrt{1-\mu})$ when $\mu$ tends to 1 , or $\rho \rightarrow \mathcal{O}(\sqrt{1+\mu})$ when $\mu \rightarrow-1$. The same kind of behaviour reveals the coefficients on the right-hand side of the integral of the Mazur identity Eq.(46). On the other hand, when we approach the axes of rotation $f_{\phi \phi} \simeq \mathcal{O}(1)$. Similarly, inspection of Eqs.(30)-(31) reveals that $v, a, e^{2 \phi}$ and $\kappa$ tend to $\mathcal{O}(1)$ as we approach the considered limit. 
It remains to take into account the behaviour of the deviation matrix near the black hole event horizon. From the previous considerations one has that if $\lambda \rightarrow c f_{\phi \phi} \simeq \mathcal{O}(1)$ and $\rho \rightarrow \mathcal{O}(\sqrt{\lambda-c})$. The inspection of the behaviour of the fields in EMAD-gravity given by the relations (30) and (31) enables to conclude that as $\lambda \rightarrow c$ they are proportional to $\mathcal{O}(1)$. They are well-behaved functions as $\lambda$ tends to the constant value $c$. The same behaviours reveal the coefficients in relation (46). Summing it all up, we draw the conclusion that it is sufficient to establish, that $\operatorname{tr} \Psi \simeq \mathcal{O}(1)$ near the event horizon of Kerr-Sen black hole.

On the other hand, at spatial infinity $f_{\phi \phi} \simeq \mathcal{O}(\lambda)$ and the coefficients in the Mazur integral given by Eq. (46) are of the same form. The same tendency is provided by $\rho$. Then, the careful inspection of all the components building the trace of the deviation matrix $\Psi$ reveals that $\partial_{\mu}(\operatorname{tr} \Psi) \simeq \mathcal{O}\left(\lambda^{-4}\right)$ plus terms of order $\lambda^{n}$, where $n>-4$.

Just we have shown that $\operatorname{tr} \Psi$ is bounded in the orbit space and vanishing at spatial infinity. It concludes that two configurations conditions coincide, i.e., $M_{[0]}=M_{[1]}$ in all the domain of outer communication $<<\mathcal{J}>>$. It provides that two solutions of equations of motion for EMAD-gravity underlying the same boundary and regularity conditions coincide with each other for at least one point in $\langle<\mathcal{J}\rangle\rangle$. We can assert to the coclusion.

\section{Theorem:}

Let us consider a stationary axisymmetric solution to four-dimensional EMAD-gravity being the low-energy limit of the heterotic string theory with asymptotically timelike Killing vector field $k_{\mu}$ and spacelike Killing vector field $\phi_{\mu}$ responsible for rotation. Then, any solution with the same boundary and regularity conditions as the Kerr-Sen black hole is the Kerr-Sen solution itself.

\section{UNIQUENESS OF EXTREMAL KERR-SEN SOLUTION}

Consider now, the extremal Kerr-Sen solution, i.e., $\hat{r}_{+}=\hat{r}_{-}$. Our next task will be to extract the near-horizon geometry of the extremal solution. To proceed further, let us define the coordinate transformation in the form as follows:

$$
\begin{aligned}
\hat{t} & =2 M \frac{\left(M-\frac{r_{-}}{2}\right)}{\left(M+\frac{r_{-}}{2}\right) \lambda} t, \\
\hat{\phi} & =\phi+\frac{\left(M-\frac{r_{-}}{2}\right)}{\left(M+\frac{r_{-}}{2}\right) \lambda} \hat{t}, \\
\hat{r} & =\frac{\lambda}{y}\left(M+\frac{r_{-}}{2}\right)+\left(M+\frac{r_{-}}{2}\right) .
\end{aligned}
$$

Taking the limit of the scaling parameter $\lambda \rightarrow 0$, we arrive at the near-horizon geometry of the Kerr-Sen extremal black hole spacetime. Consequently, we obtain the metric which yields

$$
\begin{aligned}
d s^{2} & =\left(M^{2}\left(1+\cos ^{2} \theta\right)-r_{-} M \cos ^{2} \theta-\frac{r_{-}^{2}}{4} \sin ^{2} \theta\right)\left(-\frac{d t^{2}+d y^{2}}{y^{2}}+d \theta^{2}\right) \\
& +\frac{4 \sin ^{2} \theta M^{2}\left(M-\frac{r_{-}}{2}\right)^{2}}{\left(M^{2}\left(1+\cos ^{2} \theta\right)-r_{-} M \cos ^{2} \theta-\frac{r_{-}^{2}}{4} \sin ^{2} \theta\right)}\left(d \phi+\frac{d t}{y}\right)^{2} .
\end{aligned}
$$

In order to put the near-horizon metric for the Kerr-Sen extremal black hole into the Weyl-Papapetrou form, one can remind that $(y, \theta)$ part of the line element is conformal to $d y^{2}+y^{2} d \theta^{2}$. Thus, let us introduce the change of variables given by

$$
\rho=y \sin \theta, \quad z=y \cos \theta .
$$

Using the above relation (53) the line element (52) takes the form of (2).

For the near-horizon geometry it turns out that the fields appearing in EMAD-gravity can be determined by

$$
\begin{aligned}
v & =\frac{\left(2 M r_{-}\right)^{\frac{1}{2}}\left(M-\frac{r_{-}}{2}\right)}{M^{2}\left(1+\cos ^{2} \theta\right)-\frac{r_{-}^{2}}{4} \sin ^{2} \theta-M r_{-} \cos ^{2} \theta}, \\
\kappa & =-\frac{\left(2 M r_{-}\right)^{\frac{1}{2}}\left(M-\frac{r_{-}}{2}\right) \cos \theta}{M^{2}\left(1+\cos ^{2} \theta\right)-\frac{r_{-}^{2}}{4} \sin ^{2} \theta-M r_{-} \cos ^{2} \theta},
\end{aligned}
$$




$$
\begin{aligned}
a & =\frac{r_{-} \cos \theta}{\left(M-\frac{r_{-}}{2}\right)\left(1+\cos ^{2} \theta\right)}, \\
e^{2 \phi} & =\frac{\left(M-\frac{r_{-}}{2}\right)\left(1+\cos ^{2} \theta\right)}{M^{2}\left(1+\cos ^{2} \theta\right)-\frac{r_{-}^{2}}{4} \sin ^{2} \theta-M r_{-} \cos ^{2} \theta},
\end{aligned}
$$

On this account, the components of the current matrix are provided by the following relations:

$$
\begin{gathered}
w=\frac{\left(2 M r_{-}\right)^{\frac{1}{2}}}{M^{2}\left(1+\cos ^{2} \theta\right)-\frac{r_{-}^{2}}{4} \sin ^{2} \theta-M r_{-} \cos ^{2} \theta}\left[\left(M-\frac{r_{-}}{2}\right)\left(1+\cos ^{2} \theta\right)-r_{-}\right] \frac{\cos \theta}{1+\cos ^{2} \theta}, \\
e^{-2 \phi} v=\frac{\left(2 M r_{-}\right)^{\frac{1}{2}}}{\left(M-\frac{r_{-}}{2}\right)\left(1+\cos ^{2} \theta\right)}, \\
e^{-2 \phi} v^{2}=\frac{2 M r_{-}}{M^{2}\left(1+\cos ^{2} \theta\right)-\frac{r_{-}^{2}}{4} \sin ^{2} \theta-M r_{-} \cos ^{2} \theta},
\end{gathered}
$$

and combining relations (54) and (58) we get the following:

$$
v w=\frac{2 M r_{-}\left(M-\frac{r_{-}}{2}\right)}{\left(M^{2}\left(1+\cos ^{2} \theta\right)-\frac{r_{-}^{2}}{4} \sin ^{2} \theta-M r_{-} \cos ^{2} \theta\right)^{2}}\left[\left(M-\frac{r_{-}}{2}\right)\left(1+\cos ^{2} \theta\right)-r_{-}\right] \frac{\cos \theta}{1+\cos ^{2} \theta} .
$$

\section{A. Boundary conditions}

Now we shall study the behaviour of the all components comprising the deviation matrix $\Psi$ as one approaches near-horizon limit, axes of rotations and asymptotic spatial infinity. Inspection of Eqs.(58) and (61) reveals that they are proportional to $\frac{\cos \theta}{1+\cos ^{2} \theta}$, which can be rewritten in the form as follows

$$
\frac{\cos \theta}{1+\cos ^{2} \theta}=\frac{1}{2}-\frac{\sin ^{2} \theta}{4\left(1+\cos ^{2} \theta\right) \cos ^{2} \frac{\theta}{2}} .
$$

This shows that the deviation from the constant factors out through the term proportional to $\sin ^{2} \theta$. By virtue of the relation described by (55) one gets that

$$
\kappa \simeq \frac{\alpha}{2}-\frac{\sin ^{2} \theta}{4\left(1+\cos ^{2} \theta\right) \cos ^{2} \frac{\theta}{2}\left[M^{2}+\mathcal{O}\left(\sin ^{2} \theta\right)\right]},
$$

where $\alpha$ is a constant. Returning to the relation (56) one can reveal that it provides the same deviation from constant factor through term proportional to $\sin ^{2} \theta$. On the other hand, the dilaton term reduces to the following form:

$$
e^{2 \phi} \simeq \frac{\left(M-\frac{r_{-}}{2}\right)^{2}}{M^{2}+\mathcal{O}\left(\sin ^{2} \theta\right)} \simeq \mathcal{O}(1)
$$

Let us consider the behaviour of the deviation matrix $\Psi$ near the event horizon of the extremal Kerr-Sen solution given by the line element (52). Using Eqs.(58)-(64) one can calculate the trace of $\Psi$. It is rather tedious work but can be easily automated by using the symbolic math program, as in the nodegenerate case. For $\theta \neq 0, \pi$ the value of $\Psi$ is finite in the limit $1 / y$, when one approaches the extremal event horizon. As we approach to the horizon for all $\theta$ including 0 and $\pi$ the value of all the ingredients in $\Phi_{A}$ are bounded and we get $\operatorname{tr} \Psi=\mathcal{O}(1)$.

Next, we shall take into consideration the asymptotic spatial infinity. We assume that the solutions are asymptotically flat, i.e., the metric coefficients have the forms of (28) and for the field under consideration relations (29) are satisfied. Inspection of all the components of the deviation matrix provides the conclusion that $\operatorname{tr} \Psi=\mathcal{O}\left(1 / \hat{r}^{2}\right)$. The same behaviour was revealed in the in the non-extremal case.

As far as the boundary conditions near the axis of rotation are concerned, they are chosen to guarantee the regularity of the line describing extremal black hole near the fixed points of rotational symmetry described by the adequate Killing vector field. As one approaches the extremal horizon along the axis $\theta=0$ and performs the necessary 
calculations, we can draw the conclusion that $\operatorname{tr} \Psi=\mathcal{O}(1)$. All these conclude the following uniqueness result: Theorem:

Let us consider a stationary axisymmetric spacetime with a connected degenerate future event horizon in fourdimensional EMAD gravity being the low-energy limit of the heterotic string theory Then, any solution with the same boundary and regularity conditions as the extremal Kerr-Sen black hole is the extremal Kerr-Sen solution itself.

\section{Acknowledgments}

This work was partially financed by the Polish budget funds in 2010 year as the research project.

[1] W.Israel, Phys. Rev. 164, 1776 (1967).

[2] W.Israel, Commun. Math. Phys. 8, 245 (1968).

[3] H.Müller zum Hagen, C.D.Robinson, and H.J.Seifert, Gen. Rel. Grav. 4, 53 (1973), H.Müller zum Hagen, C.D.Robinson, and H.J.Seifert, Gen. Rel. Grav. 5, 61 (1974)

[4] C.D.Robinson, Gen. Rel. Grav. 8, 65 (1977).

[5] R.Schoen R and S-T.Yau, Commun. Math. Phys. 65, 45 (1979).

[6] E.Witten, Commun. Math. Phys. 80, 381 (1981).

[7] G.L.Bunting and A.K.M.Masood-ul-Alam, Gen. Rel. Grav. 19, 147 (1987).

[8] P.Ruback, Class. Quantum Grav. 5, L155 (1988).

[9] A.K.M.Masood-ul-Alam, Class. Quantum Grav. 9, L53 (1992).

[10] M.Heusler, Class. Quantum Grav. 11, L49 (1994).

[11] P.T.Chruściel, Class. Quantum Grav. 16, 661 (1999).

[12] P.T.Chruściel, Class. Quantum Grav. 16, 689 (1999).

[13] P.T.Chruściel and P.Tod, Commun. Math. Phys. 271, 577 (2007).

[14] B.Carter, in Black Holes, edited by DeWitt C and DeWitt B.S (Gordon and Breach, New York) (1973).

[15] B.Carter, in Gravitation and Astrophysics, edited by B.Carter and J.B.Hartle (Plenum Press, New York) (1987).

[16] C.D.Robinson, Phys. Rev. Lett. 34, 905 (1975).

[17] P.O.Mazur, J. Phys. A: Math. Gen. 15, 3173 (1982).

[18] P.O.Mazur, Black Hole Uniqueness Theorems Preprint, hep-th 0101012 (2001).

[19] G.L.Bunting, PHD thesis, Univ.of New England, Armidale N.S.W. (1983).

[20] B.Carter, Commun. Math. Phys. 99, 565 (1985).

[21] M.Heusler, Black Hole Uniqueness Theorems (Cambridge: Cambridge University Press) (1997).

[22] D.Youm, Phys. Reports 316, 1 (1999).

[23] A.Sen, Phys. Rev. Lett. 69, 1006 (1992).

[24] D.Gal'tsov and O.Kechkin, Phys. Rev. D 50, 7394 (1994).

[25] D.Gal'tsov and O.Kechkin, Phys. Rev. Lett. 74, 2863 (1995).

[26] D.Gal'tsov and O.Kechkin, Phys. Lett. B 361, 52 (1995).

[27] D.Gal'tsov, A.Garcia, O.Kechkin, J. Math. Phys. 36, 5023 (1995).

[28] D.Gal'tsov and O.Kechkin, Phys. Rev. D 54, 1656 (1996).

[29] D.Gal'tsov and O.Kechkin, Hidden Symmetries in Dilaton-Axion Gravity, gr-qc 9606014 (1996).

[30] D.Gal'tsov and S.A.Sharakin, Phys. Lett. B 399, 250 (1997).

[31] M.Yurova, Phys. Rev. D 64, 024002 (2001).

[32] M.Yurova, Phys. Rev. D 65, 024024 (2001).

[33] T.Matos, G.Miranda, R.Sanchez-Sanchez, and P.Wiederhold, Phys. Rev. D 79, 124016 (2009).

[34] A.K.M.Masood-ul-Alam, Class. Quantum Grav. 10, 2649 (1993).

[35] M.Gürses and E.Sermutlu, Class. Quantum Grav. 12, 2799 (1995).

[36] M.Rogatko, Class. Quantum Grav. 14, 2425 (1997).

[37] M.Rogatko, Phys. Rev. D 58, 044011 (1998).

[38] M.Rogatko, Phys. Rev. D 59, 104010 (1999).

[39] M.Mars and W.Simon, Adv. Theor. Math. Phys. 6, 279 (2003).

[40] W.Simon, Lett. Math. Phys. 50, 275 (1999).

[41] H.K.Kunduri and J.Lucietti, J. Math. Phys. 50, 082502 (2009), H.K.Kunduri and J.Lucietti, Class. Quantum Grav. 26, 055019 (2009).

[42] P.Figueras and J.Lucietti, Class. Quantum Grav. 27, 095001 (2010).

[43] A.J.Amsel, G.T.Horowitz, D.Marolf, and M.M.Roberts, Phys. Rev. D 81, 024033 (2010).

[44] R.Meinel, M.Ansorg, A.Kleinwachter, G.Neugebauer, and D.Petroff, Relativistic Figure of Equilibrium, (Cambridge University Press, Cambridge, England, 2008), G.Neugebauer and R.Meinel, J. Math. Phys. 44, 3407 (2003). 
[45] P.T.Chrusciel and L.Nguyen, A Uniqueness Theorem for Degenerate Kerr-Newman Blach Holes, gr-qc 1002.1737 (2010).

[46] W.Israel and G.A.Wilson, J. Math. Phys. 13, 865 (1972).

[47] Y.Morisawa and D.Ida, Phys. Rev. D 69, 124005 (2004).

[48] Y.Morisawa, S.Tomizawa, and Y.Yasui, Phys. Rev. D 77, 064019 (2008). 